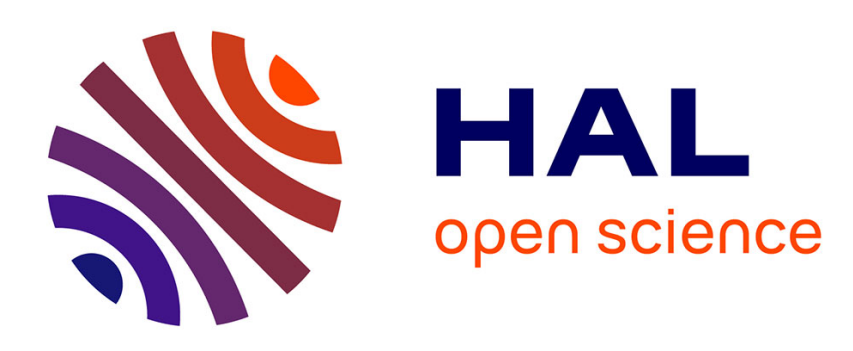

\title{
Study of Probabilistic Worst Case Inter-Beacon Delays Under Realistic Vehicular Mobility Conditions
}

Alexandre Mouradian

\section{To cite this version:}

Alexandre Mouradian. Study of Probabilistic Worst Case Inter-Beacon Delays Under Realistic Vehicular Mobility Conditions. ADHOC-NOW 2015, Jun 2015, Athens, Greece. 10.1007/978-3-319-196626_27. hal-01196312

\section{HAL Id: hal-01196312 \\ https://hal.science/hal-01196312}

Submitted on 9 Sep 2015

HAL is a multi-disciplinary open access archive for the deposit and dissemination of scientific research documents, whether they are published or not. The documents may come from teaching and research institutions in France or abroad, or from public or private research centers.
L'archive ouverte pluridisciplinaire HAL, est destinée au dépôt et à la diffusion de documents scientifiques de niveau recherche, publiés ou non, émanant des établissements d'enseignement et de recherche français ou étrangers, des laboratoires publics ou privés. 


\title{
Study of Probabilistic Worst Case Inter-Beacon Delays Under Realistic Vehicular Mobility Conditions
}

\author{
Alexandre Mouradian \\ Laboratoire des Signaux et Systèmes (L2S, UMR8506), Université Paris \\ Sud-CNRS-CentraleSupélec, F-91192 Gif-sur-Yvette \\ alexandre.mouradian@u-psud.fr
}

\begin{abstract}
Road safety applications are one of the main incentives to deploy vehicular networks. These applications rely on periodic message exchange among vehicles (known as beaconing). The beacon messages contain information about the environment which is used to perceive dangerous situations and alert the drivers. The inter-beacon delay is the time between two consecutive beacons received from a car. It is an essential parameter because, if this delay exceeds the application requirement, the application cannot accurately predict dangerous situations and alert the drivers on time. The worst case inter-beacon delay has thus to be bounded according to the application requirements. Unfortunately, a tight and strict bound is in fact very difficult to obtain for a real network because of the randomness of the collisions among beacons coming from: the unpredictable mobility patterns, random interferences, randomness of the MAC layer backoff, etc.

In this paper, we propose to provide a probabilistic worst-case of the inter-beacon delay under realistic mobility using Extreme Value Theory (EVT). EVT provides statistical tools which allow to make predictions on extreme deviations from the average of a parameter. These statistical predictions can be made based on data gathered from simulation or experimentation. We first introduce the EVT technique. Then we discuss its application to the study of inter-beacon delays. Finally, we apply EVT on the results of extensive vehicular network simulation using a realistic mobility trace: the Cologne trace.
\end{abstract}

\section{Introduction}

The road safety applications are seen as an essential motivation for the deployment of Vehicular Ad hoc NETworks (VANETs) [9]. The main goal of these applications is to avoid car crashes and thus reduce the number of road traffic deaths and injuries. We can cite as examples of safety applications [5]:

- traffic signal violation: alert neighbor cars when a user does not stop at a red traffic light;

- electronic brake: alert neighbor vehicles when a car performs an emergency braking; 
- on-coming traffic warning: notify the driver of on-coming traffic during overtaking maneuvers.

The main building block for safety applications is the exchange of periodical one-hop broadcast messages among neighbor vehicles [9]. These messages are called beacons or heartbeat messages and contain, at least, information on the car positions, directions and speeds. In the rest of this paper, as in [19] [17] we refer to these messages as beacons. Each safety application uses these beacons to build a representation of its environment, analyze the current situation and predict dangerous situations. The period, latency and scope (or range) of the beacons depend on the requirements of the application. For example, according to [9] the electronic brake application needs a beacon frequency of $10 \mathrm{~Hz}$, a maximum latency of $100 \mathrm{~ms}$ and a range of $200 \mathrm{~m}$ to be effective. In this paper, we focus on the period of these messages: the inter-beacon delay. We are thus interested in the capacity of the MAC layer to broadcast these beacons on a periodical basis which complies with the application requirements. We consider the MAC from the IEEE 802.11p standard, since it is regarded as the standard of choice for VANETs [9].

In order to be effective, critical road safety applications need strong timing guaranties on the delivery of beacons. It is thus of paramount importance that the MAC layer is able to provide worst case inter-beacon delays which are bounded. But more importantly, the bound has to be known and has to correspond to the target application. Nevertheless, most of the performance evaluation techniques of the literature such as stochastic models, simulation and experimentation focus on parameter averages [10] and give very few insights on extreme cases. This is the case, in particular, concerning many performance studies of beaconing in vehicular networks [9] [19] [17]. On the other hand, a strict bound on the inter-beacon delay is difficult, if not impossible, to obtain because of the random nature of the beacon collisions which comes from the mobility patterns, interferences, pathloss, randomness of the MAC layer backoff, etc.

In this paper, we propose to estimate a probabilistic bound on the interbeacon delay using statistical tools. The probabilistic worst case delay can be viewed as the probability that the maximum delay is less than a given value. This quantity is very useful for the system designer which must ensure that the system can handle the timing requirements of the application with a high probability. An advantage of the statistical approach is that it allows to evaluate directly the real studied system (or a highly detailed simulation model) instead of working on an abstract theoretical model.

The statistical tools we use come from Extreme Value Theory (EVT) which is presented in sections 3 . The method can be summed up in the three following steps: (1) produce/Gather data (by simulation or experimentation); (2) extract extreme values from the data; (3) fit extracted values to a probability distribution predicted by the theory.

The main contribution of this paper is to use EVT to characterize the distribution of high inter-beacon delays when using the IEEE 802.11p standard under realistic mobility conditions. We first discuss EVT applicability to study 
inter-beacon delays and then use it on simulation results. The simulations are performed in ns2 [1] using a highly realistic mobility trace developed by Uppor and Fiore [21]. Up to our knowledge, this study represents the first application of EVT to the study of extreme delays in VANETs and is the first characterization of extreme inter-beacon delays under realistic mobility conditions using the IEEE $802.11 p$ standard.

The remainder of the paper is organized as follows. In section 2 the related work about safety beaconing performance and EVT is presented and commented. In section 3, we introduce EVT main theorems and discuss the application of EVT to the study of large inter-beacon delays. In section 4, we present the simulation setup and the produced data sets. In section 5 we describe the results of the application of EVT to inter-beacon delay simulation data and provide discussion about the method and the obtained probabilistic worst-case interbeacon delays. Section 6 gives the conclusion remarks and lists future works.

\section{Related work}

In this section we present the related work regarding the study of beaconing in VANETs with 802.11p, and on the application of EVT in general and more specifically in computer science.

Many studies concerning the performance of beaconing using 802.11p exist in the literature [9] [19] [11] [3] [17] [16]. In [9], the authors study the performance of beaconing in VANETs through simulation. They use the ns2 simulator. In their scenarios, the vehicles are positioned on parallel lines (straight highway scenario) and are not moving. The authors provide the probability of a successful beacon reception for different beaconing frequencies. The results show that the probability of reception decreases rapidly after few hundred meters between the emitter and receiver. In [19], Stanica et al. propose an analytical model of the probability of collision and the reception probability of beacons. They highlight the low reliability of beaconing with 802.11 p as with their model, the reception probability is never greater than 0.7 . They confirm their findings through simulation. The authors also show that the performance depends on the 802.11 contention window and derive its optimal value. In [16], the repartition of the nodes of the VANET is modeled as a Poisson Point Process. The authors show that above a critical density of nodes, the CSMA scheme of $802.11 \mathrm{p}$ behaves like an ALOHA protocol and its performance is thus highly deteriorated. The authors study the reception probability for simple highway scenarios under various traffic conditions and transmission power hypotheses. Again the reliability of beaconing is very low. The authors of [17] simulate beaconing using ns2 and the realistic Cologne mobility trace [21]. They study the probability of beacon delivery in different areas of the city. They confirm the bad reliability of $802.11 \mathrm{p}$ in a realistic mobility scenario. Despite the fact that many studies evaluate the reliability of beaconing with 802.11 p, none of the presented study provide insight on the worst case inter-beacon delay under realistic mobility conditions (we can note that [11] is concerned about the inter-beacon delay distribution, but not 
the worst case). Yet, this parameter is of paramount importance to evaluate the achievable timeliness of safety applications.

In the literature, EVT has been extensively used in various contexts: extreme rainfalls [18], forest fires [2], wind speed [4], financial crashes [8] studies. EVT has also been used in the context of computer networks for the estimation of traffic peaks or bursts [13] [20] [6]. In [20], the author proposes to study the traffic on an Ethernet network in order to predict traffic peaks. The author shows that the gathered extreme deviations fit very well to a Generalized Pareto distribution as predicted by the theory. In another study [13], the authors apply EVT to the study of traffic throughput in wireless networks and show that the generalized EVT distribution is a better match for large deviation prediction than exponential, gamma or log-normal distributions. In [6] the authors fit Ethernet traffic throughput data to a Weibull distribution (also predicted by EVT). Nevertheless, these applications of EVT focus on network traffic throughput. Here we are more interested in the possibility to use such technique to study large delays in computer networks.

In order to find applications of EVT to the study of large delays in computer science, we have to look to the field of worst case execution times. Works such as [7] [14] make use of EVT to derive probabilistic worst case execution times. In our work, we explore the case of inter-beacon delays instead of task executions. We comment, in section 3 and 5, the issues which may arise when applying EVT to this specific case.

The novelty of our work is twofold: we are interested in characterizing large inter-beacon delays behavior instead of their probability of reception in realistic mobility conditions, and we use the EVT method which up to our knowledge have never been apply to the study of delays in networks.

\section{Extreme Value Theory applied to delays in networks}

\subsection{A brief introduction to EVT}

Extreme Value Theory has been developed during the 20th century and is now a well established tool to study extreme deviations from the average of a measured phenomenon [4]. EVT is built around two main theorems: the FisherTippett-Gnedenko theorem and the Pickands-Balkema-de Haan theorem. As we will detail below, the former is interested in the maximum value of a sequence of variables, whereas the latter focuses on the values of a sequence which are above a given threshold.

The Fisher-Tippett-Gnedenko theorem states that given $\left\{X_{1}, \ldots, X_{n}\right\}$ a sequence of independent and identically distributed (i.i.d.) variables, the distribution of $M_{n}=\max \left\{X_{1}, \ldots, X_{n}\right\}$ the variable representing the maximum value of the sequence converges (for large $n$ ) toward one of these three distribution families characterized by their CDF (Cumulative Distribution Function):

- Fréchet:

$$
G(x)=e^{-\left(\frac{x-m}{s}\right)^{-\alpha}}, \text { for } x>m
$$


if the distribution has a heavy tail;

- Gumbel:

$$
G(x)=e^{-e^{-\frac{x-m}{s}}}
$$

if the distribution has an exponential tail;

- Weibull

$$
G(x)=e^{-\left(-\left(\frac{x-m}{s}\right)\right)^{\alpha}}, \text { for } x<m
$$

if the distribution has a finite maximum;

with $m, s$ and $\alpha$ the distribution parameters and $\alpha>0$ in all cases.

The second theorem of EVT is the Pickands-Balkema-de Haan theorem. It states that given $\left\{X_{1}, \ldots, X_{n}\right\}$ a sequence of i.i.d. variables, their conditional distribution $F_{u}(y)=P(X-u<y \mid X>u)$ converges toward a generalized Pareto distribution for large $u$ :

$$
\left\{\begin{array}{l}
G(y)=1-\left(1+\frac{(y-m) \gamma}{s}\right)^{-\frac{1}{\gamma}}, \text { if } \gamma \neq 0 \\
G(y)=1-e^{-\frac{y-m}{s}}, \text { if } \gamma=0
\end{array}\right.
$$

To each theorem corresponds a method which can be applied to characterize the distributions of extreme variation of a phenomenon. For the first theorem the method is the Block Maxima (BM) method in which the sequence of measured data is divided into blocks and the maximum of each block is computed. The maxima are then fitted to one of the three previously mentioned distributions. The second theorem of EVT corresponds to the Peak Over Threshold (POT) method. In this method, a threshold value is chosen and the data points which are above the threshold are collected and fitted to a generalized Pareto distribution.

\subsection{Application to the study of inter-beacon delays}

According to Fisher-Tippett-Gnedenko and the Pickands-Balkema-de Haan theorems, to apply EVT, the sequence of variables must be independent and identically distributed. The question then is: can it be the case for inter-beacon delays in VANETs?

Let's first consider two nodes A and B. Can we assume that their interbeacon delays are identically distributed? We would tend to answer yes if the network environment and measure conditions are the same for both $\mathrm{A}$ and $\mathrm{B}$ : same channel conditions, same node density, fair access to the medium, etc. Because in this case the packet collisions and packet loss inducing inter-beacon delays would be identically distributed. This leads us to believe that to apply EVT on inter-beacon delays we have to be careful not to have great disparities in the studied network like very dense and very sparse areas in the same network. This statement will be verified in section 5 .

Concerning the independence hypothesis, we can argue that it will not always be true in the case of inter-beacon delays. If we consider the successive interbeacon delays between two nodes with bad channel conditions, they will tend to be correlated (if the channel conditions are stable between two beacons). 
Nevertheless, in the literature, we find many EVT application cases on data sequences which seem correlated in time. For instance, in [4] it is mentioned that it is possible to fit very accurately maximum wind speeds to an extreme value distribution. Nevertheless, wind speed measures may appear to be temporally correlated as studied in [12]. This shows that EVT may accurately model the large values of a phenomenon even if the measures are partially correlated.

It is not clear from the literature, what are the cases where EVT can or cannot be applied. In section 5, we will consider the i.i.d hypothesis true, try to apply EVT and evaluate how the data fits the model (the fit can be assessed by a statistical test as explained in section 3.4). We will then discuss in which cases EVT is meaningful.

Due to the lack of space, in this paper, we consider only the application of the Fisher-Tippett-Gnedenko theorem. The second theorem of EVT is left as a future work.

\subsection{Gathering and arranging data for EVT}

The first element needed in order to apply EVT is a set of data which is a realization of the sequence of random variables $\left\{X_{1}, \ldots, X_{n}\right\}$ mentioned in the previous section. In our case it will be measures of inter-beacon delays obtained from simulation. In this paper, we consider technique associated with the FisherTippett-Gnedenko theorem to process the data. This technique is known as Block Maxima (BM). The principle of the BM technique is to divide the data sequence into blocks and to take the maximum of each block. In the literature, a block is often defined as a time interval [4]. According to the Fisher-TippettGnedenko theorem the sequence of block maxima must converge to an extreme value distribution. The observed sequence of block maxima can thus be fitted to one of the Gumbel, Weibull or Fréchet distributions. The type of distribution the data will converge to is difficult to predict from the raw data. In section 5 we thus try to fit the data to the three considered distribution and discuss which is the most accurate model for the worst-case inter-beacon delay.

\subsection{Fitting technique and statistical test}

In the previous subsection, we have described how to retrieve extreme value data from the original data set. We then have to fit this extreme value data to one of the extreme value distributions predicted by EVT and assess that the fitted distribution is an acceptable representation of the data thanks to a goodness-of-fit statistical test. In this paper we use the Maximum Likelihood Estimation (MLE) technique to estimate the parameters of the distribution, and the Pearson's chi-squared test to assess the goodness-of-fit.

The MLE technique is based on the likelihood function, which is defined as follows:

$$
L(\mathbf{x}, \theta)=\prod_{i=1}^{n} f\left(x_{i}, \theta\right)
$$


with $\mathbf{x}$ a vector of observed values, $f$ the pdf we want to fit to the values and $\theta$ the vector of parameters for the pdf.

The MLE method consists in finding a vector of parameters $\theta$ such that $L(\mathbf{x}, \theta)$ is maximized. In practice, for the problem to be tractable, it is actually the logarithm of the likelihood which is maximized (the logarithm function preserves the optimum). In this paper, in order to solve this optimization problem we use the Nelder-Mead method [15].

Once the optimal distribution parameters are obtained, we have to verify that the fitted distribution is actually a convincing representation for the data. For that purpose, we use two tools: the Pearson chi-squared test and quantilequantile plots [10]. The former is a statistical test which assesses if there is a statistical difference between an observed data frequency distribution and a theoretical distribution. We can note that this test statistic offers a quality indicator for the fit which can be used in the case several distributions pass the chi-squared test as in [18].

The quantile-quantile plot [10] (or Q-Q plot) is a tool which allows to graphically compare two distributions. In our case, it consists in plotting the quantiles of the collected data against the quantiles of the fitted distribution. If the two distributions match, we should obtain the relation $x=y$. As described in [4] and [10], the plot consists in a set of points $(x, y)$ where $x \in x_{1}, \ldots, x_{n}$ with $x_{1} \ldots x_{n}$ the ordered set of data points (in increasing order) and $y=F^{-1}\left(\frac{i}{n+1}\right)$ with $n$ the number of data points, $i=1, \ldots, n$ and $F^{-1}$ the inverse CDF of the fitted distribution (the inverse CDF corresponds to the quantile function).

\section{Simulation}

In this section we present the simulation parameters and the obtained data sets on which we apply EVT.

\subsection{Simulation setup}

We use the discrete-event simulator ns2 [1] to perform the simulations, the main parameters are described in Table 1 . The simulation setup is actually quite classic, we thus focus on the description of the realistic mobility trace and how we use it in the simulator.

The mobility trace we use in the simulations is a realistic micro-mobility trace of the city of Cologne generated by Uppoor and Fiore [21]. The trace covers a 400 $\mathrm{km}^{2}$ area and a period of 24 hours and contains about 700000 vehicle travels. In this paper we use the part of the trace available online which covers the $6 \mathrm{am}-8 \mathrm{am}$ period. In Figure 1, we plot the instantaneous positions of the nodes at $8 \mathrm{am}$. Each point corresponds to a vehicle position in the coordinate system provided by the trace (the coordinates are expressed in meters). The trace from $6 \mathrm{am}$ to $8 \mathrm{am}$ contains more than 300000 different vehicles, it is thus not possible to simulate the whole scenario in ns2. As we are interested in local communications (onehop broadcasts), we decide to divide the network and restrict the simulations to 


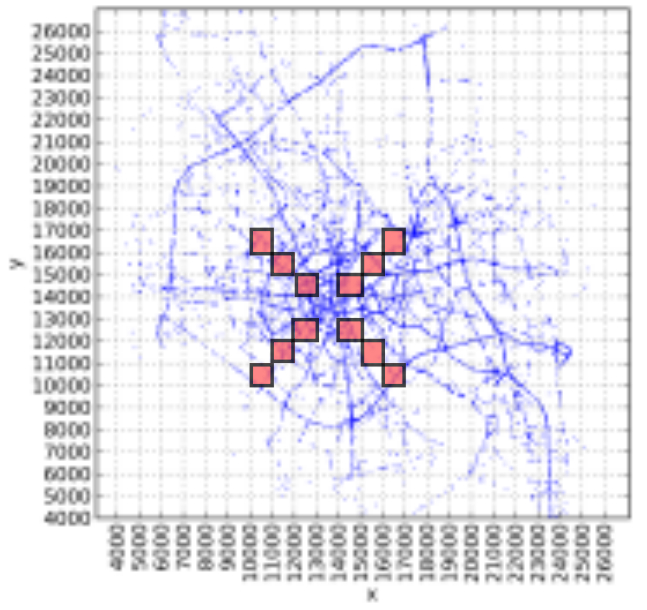

Fig. 1: Snapshot of the Cologne data set

1000x1000m squares. The considered squares are highlighted in Figure 1. They contain different types of road traffic (fluid traffic, traffic jams, etc) and different vehicle densities. Even with this space division of the trace, the number of cars in one square can reach several hundreds and, in some cases, the simulations are either very long (dozens of hours) or not possible (the memory of the machine used for the simulation is not sufficient). We thus divide again the trace, but this time into time intervals. Instead of having 2 hours of simulated time we produce subset traces of 200 seconds. Each of the time blocks also contains different traffic conditions, since the traffic changes over time in the trace.

In each simulation, we monitor the inter-beacon delay as well as the emitterreceiver distance. A simulation typically provides around 2.5 millions measurements of inter-beacon delays (it varies depending on the number of cars present during the simulation).

\begin{tabular}{|l|l|}
\hline Parameter & Value \\
\hline Bitrate & $6 \mathrm{Mbps}$ \\
Transmission power & $10 \mathrm{dBm}$ \\
Simulation area & $1000 \times 1000 \mathrm{~m}$ \\
Beacon size & 400 bytes \\
MAC and Phy & $802.11 \mathrm{p}$ \\
Propagation model & Nakagami m=1 \\
Beacon frequency & $10 \mathrm{~Hz}$ \\
\hline
\end{tabular}

Table 1: Simulation parameters. 


\subsection{Obtained data sets}

For the BM technique, we need to divide the data into blocks and retrieve the maximum of each block. We choose to use three different ways of producing the data which is then fitted to EVT distributions:

1. The first one consists in taking the maximum of each block of 200 seconds (corresponding to one run of the simulator) for the whole trace duration and all the highlighted squares in Figure 1.

2. In the second one we take only one square and one portion of time and re-run the simulation for that particular square and block several times.

3. The third case is the same as the second but we change the order of the beacon start of the nodes at each run (in the second case, the order of beacon start dates is generated randomly once and the same order is repeated in every simulation).

These different setups allow us to understand how disparities in the simulation data affects the applicability of the BM method. Indeed, in the first case, the data collected comes from various situations in terms of car traffic amount, network density, etc. Whereas in the two last cases the blocks are more similar to one another (in the second case there are more correlations between the runs because the beacon start dates are the same).

For setup 1, we run simulations for 13 successive blocks of 200 seconds for each of the 12 considered squares. We take the maximum inter-beacon delay for each run and thus obtain 156 values. For setup 2 and 3 we run 300 simulation of the considered block, so we obtain 300 maximum values. In the results presented in the following sections, we first consider the receivers in a 500 meters range from the sender, and we then observe the impact of the range on the maximum delay distribution.

\section{Results and discussion}

In this section we present the results of the application of the EVT method presented in section 3 to the data sets presented in section 4.2.

Figure $2 \mathrm{a}$ is a histogram representation of the maximum delays for the first data set. First, we have to note that the measured maximum inter-beacon delays are very large compared to the inter-beacon emission period ( 0.1 seconds). This is due to two main reasons: the mobility and the broadcast scheme used. When a node broadcasts its beacon using 802.11 p, it cannot detect collisions. Indeed, collisions happen at the level of the receivers and the sender does not know which of its neighbors will actually receive the packet and does not wait for acknowledgments in the case of broadcasting. Moreover, broadcast messages are subject to the hidden terminal problem since the RTS/CTS messages are not used for broadcast. These problems have been highlighted several times in the literature [9] [19] [16]. In order to better comprehend the observed long delays, let's consider the following scenario: first, a node A periodically receives a beacon 

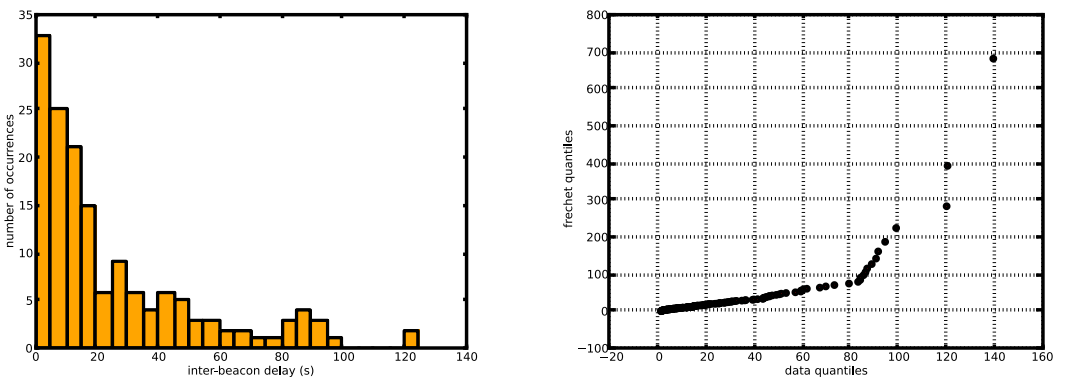

(a) Histogram of the maximum inter-beacon(b) QQ-plot: data against Fréchet distribudelays tion
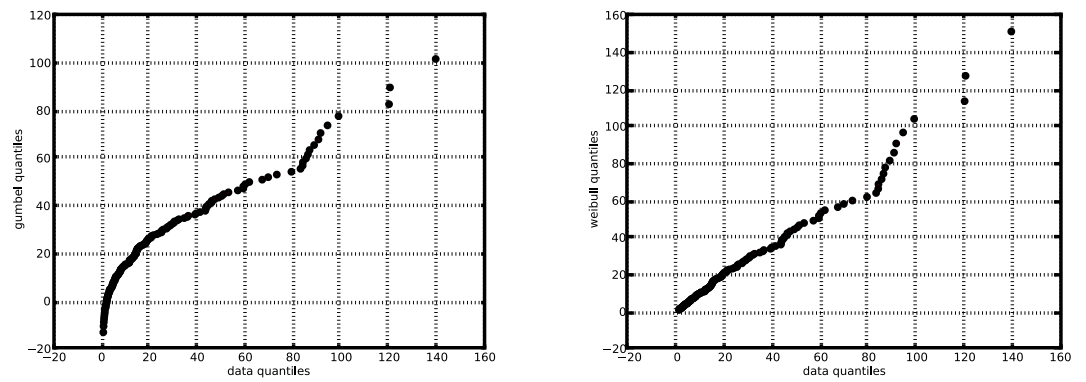

(c) QQ-plot: data against Gumbel distribu-(d) QQ-plot: data against Weibull distribution tion

Fig. 2: Results for all the squares and blocks and 500m range

from one of its neighbors $\mathrm{B}$, then another node $\mathrm{C}$ moves in the neighborhood of $\mathrm{A}$ and the beacons from $\mathrm{C}$ collide with those of $\mathrm{B}$ (in the case of the hidden terminal problem the beacons may constantly collide), then $\mathrm{C}$ moves out of range of $\mathrm{A}$ again. In this scenario, when $\mathrm{C}$ at last get out of the range of $\mathrm{A}$, it puts an end to the collisions with beacons from $\mathrm{B}$. When $\mathrm{A}$ receives the first beacon from $\mathrm{B}$ after $\mathrm{C}$ went out, the inter-beacon delay is approximately equal to the duration of the presence of $\mathrm{C}$ in the range of $\mathrm{A}$. In the cologne trace case, this delay can be of the order of tens of seconds as we observe in the simulations results.

Let's now focus on the fitting of the data of the first data set to the different extreme value distribution families. Figures $2 \mathrm{~b}, 2 \mathrm{c}$ and $2 \mathrm{~d}$ respectively represent the QQ-plots of the fitted Fréchet, Gumbel and Weibull distributions (fitting has been realized using the MLE method described in section 3). First we observe that none of the distribution fits well to the data. Indeed, none of the graphs 
show a $x=y$ curve. Nevertheless, we remark that in the Fréchet case the curve is piecewise linear which seems to indicate that there are linear relations between the data quantiles and the fitted Fréchet distribution quantiles. In the case of the fitted Weibull distribution, we observe that for $x$ and $y$ lesser than 60 , the points are approximately on the $x=y$ curve.

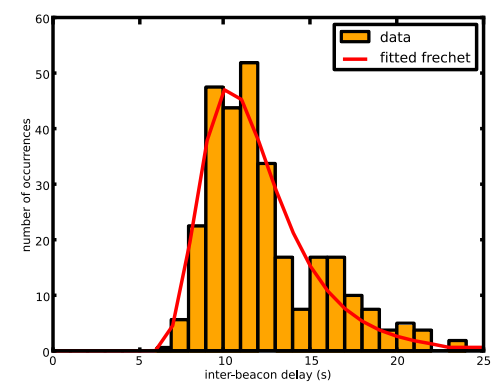

(a) Histogram of the maximum inter-beacon(b) QQ-plot: data against Fréchet distribudelays

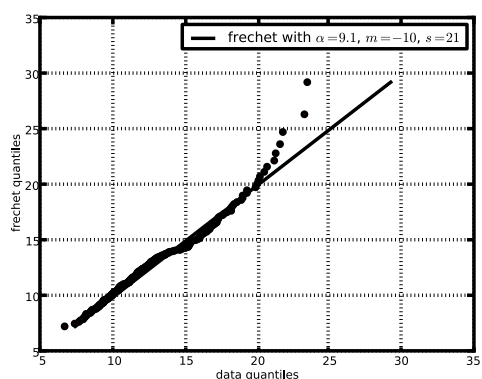

tion

Fig. 3: Results for one square during 200 seconds

The fact that the QQ-plots are piecewise, seems to indicate that the actual maximum inter-delay distribution is multi-modal. In fact, from the simulation data, we observe that the different modes correspond to different areas (squares) of the network and different time periods. We conclude that the EVT hypothesis which states that the set of inter-beacon delays are identically distributed does not hold for this data set and thus it is not possible to apply EVT. As a matter of fact, the results of the chi-squared tests for all three EVT distributions for this data set are negative (the tests are performed with a $p=0.05$ significance level).

Figures $3 \mathrm{a}$ and $3 \mathrm{~b}$ respectively depict the frequency plot of the inter-beacon delays with the expected frequencies from the fitted Fréchet distribution and the QQ-plot of the data against the fitted distribution for the second data set. As stated in section 4.2, this set consists of one square and one portion of 200 seconds run 300 times. The chosen square is defined as $x \in[11000,12000]$ and $y \in[11000,12000]$ (cf. Figure 1) and the considered time block is from 1200 to 1400 seconds of the original trace. In this case, only the fitted Fréchet distribution successfully passes the Pearson chi-squared test. The QQ-plot (Figure 3b) shows that the fit is good for the lowest values and of a lower quality for the highest values. We can also still discern at least two modes in the frequency distribution in Figure 3a (also visible in Figure 3b, because the points lie over the $x=y$ curve for $x<15$ and then under until approximately $x=18$ ). 


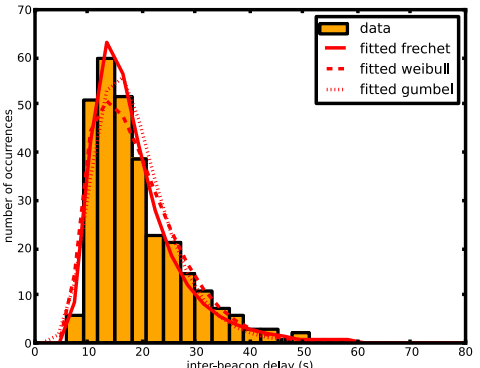

(a) Histogram of the maximum inter-beacon(b) QQ-plot: data against Fréchet distribudelays

Fig. 4: Results for one square during 200 seconds with random beacon start at each run

Figures $4 \mathrm{a}$ and $4 \mathrm{~b}$ present the results for the third simulation setup (the third data set): it is the same as the previous (one square for one 200 second interval run multiple times) but the nodes are starting their beacon emission at different dates in each simulation. The starting dates are in fact uniformly distributed in the first second of the simulation. For this data set, all three fitted EVT distributions pass the chi-squared test. Figure $4 \mathrm{a}$ depicts the frequency plot of the inter-beacon delays with the expected frequencies from the three EVT distribution. In Figure 4b, we present only the QQ-plot for the fitted Fréchet distribution because, even if all the distributions pass the chi-squared test, the Fréchet distribution is the best fit (the one with the lowest test statistic). In this QQ-plot, we observe, as in the last, that the fit is good for the lower values and less good for higher values. Nevertheless, for this data set, we do not observe multimodal tendency which seems to indicate that the identically distributed hypothesis holds. We can add that for larger blocks (more than 200 seconds) the fit is even better. Unfortunately we cannot present the results here due to the lack of space. The discussion on the choice of the size of the block for the BM method will thus be presented in future works. We can also note that the provided probabilistic bound (corresponding to the fitted Fréchet distribution) is more pessimistic in third data set case than in the second as can be seen by comparing the distributions of Figures $3 \mathrm{a}$ and $4 \mathrm{a}$. In the last case, larger inter-beacon delays are more probable.

These results show that the Fréchet distribution is an adequate model for the distribution of large inter-beacon delays in VANETs with realistic mobility. Nevertheless, the distribution models accurately model the data only if the network conditions are coherent in every locations of the network where measures are taken (as shown by the failure of EVT for data set 1). It means for example, that for different network densities different fitted Fréchet distributions apply. 


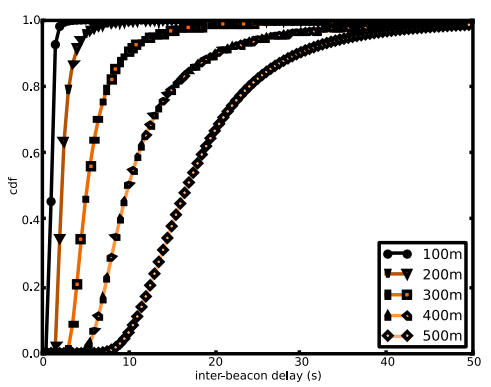

Fig. 5: CDF for different beacon ranges

All the results we have presented so far are for a 500 meters range: we compute the inter-beacon delays for receivers within 500 meters of the sender. Nevertheless, for most of critical safety applications, the range of beacon emission can be much lower [5]. In Figure 5, we plot the CDFs of fitted Fréchet distribution for different ranges. The CDF of the maximum delay expresses the probability that the maximum delay is under a given value. It corresponds to the probabilistic worst case delay. Thanks to this probabilistic worst case delay, the system designer can state that, for example, "the probability that the maximum interbeacon delay in a 100 meters range from the sender is less than 5 seconds is close to one". Whether this probabilistic delay bound is sufficient or not depends on the considered application. In Figure 5, we can notice that the CDF for higher ranges is lower. This means that the probability that the maximum delay is over a given value is higher for higher ranges. This can be explained by two phenomena: the higher probability of packet loss at longer distances and the hidden terminal problem as explained above in this section.

\section{Conclusion and future works}

In this paper, we study large inter-beacon delays in VANETs under realistic mobility with IEEE 802.11p. Understanding such delays is useful to assert if safety application requirements will be met. We show that large inter-beacon delays are Fréchet distributed. This result can be used in order to evaluate the performance of vehicular safety applications. We also show that the EVT method used to reach that conclusion is applicable for the study of delays in large scale wireless networks such as VANETs. Finally we confirm, in realistic mobility conditions, the results [19] [16] which predict the bad performance of IEEE 802.11 broadcast. In future works we plan to: apply the second theorem of EVT to the study of delays in VANETs, evaluate the impact of the choice of the block size (in the BM method) on the quality of the obtained distribution (the goodness of fit), and compare the distribution obtained from various data sets (mobility traces), to evaluate how general the EVT results are. 


\section{References}

1. The Network Simulator - ns-2. http://www.isi.edu/nsnam/ns/

2. Alvarado, E., Sandberg, D.V., Pickford, S.G.: Modeling large forest fires as extreme events. National Emergency Training Center (1998)

3. Autolitano, A., Campolo, C., Molinaro, A., Scopigno, R.M., Vesco, A.: An insight into Decentralized Congestion Control techniques for VANETs from ETSI TS 102 687 V1. 1.1. pp. 1-6. IFIP WD, Valencia, Spain (2013)

4. Beirlant, J., Goegebeur, Y., Segers, J., Teugels, J.: Statistics of extremes: theory and applications. John Wiley \& Sons (2006)

5. Consortium, C.V.S.C., et al.: Vehicle safety communications project: task 3 final report: identify intelligent vehicle safety applications enabled by dsrc. National Highway Traffic Safety Administration, US Department of Transportation, Washington DC (2005)

6. Dahab, A.Y., bin Md Said, A., bin Hasbullah, H.: Application of extreme value theory to bursts prediction. SPIJ 3(4), 55 (2009)

7. Edgar, S., Burns, A.: Statistical analysis of wcet for scheduling. pp. 215-224. IEEE RTSS, London, UK (2001)

8. Embrechts, P., Resnick, S.I., Samorodnitsky, G.: Extreme value theory as a risk management tool. North American Actuarial Journal 3(2), 30-41 (1999)

9. Hartenstein, H., Laberteaux, K.: VANET: vehicular applications and internetworking technologies. Wiley Online Library (2010)

10. Jain, R.: The art of computer systems performance analysis. John Wiley \& Sons (2008)

11. Kloiber, B., Garcia, C., Härri, J., Strang, T.: Update delay: A new informationcentric metric for a combined communication and application level reliability evaluation of cam based safety applications. In: ITS World Congress (2012)

12. Koçak, K.: Practical ways of evaluating wind speed persistence. Energy 33(1), 65 $-70(2008)$

13. Liu, C., Shu, Y., Liu, J., Yang, O.: Application of extreme value theory to the analysis of wireless network traffic. pp. 486-491. IEEE ICC, Glasgow, Scotland (2007)

14. Lu, Y., Nolte, T., Bate, I., Cucu-Grosjean, L.: A statistical response-time analysis of real-time embedded systems. pp. 351-362. IEEE RTSS, Puerto Rico (2012)

15. Nelder, J.A., Mead, R.: A simplex method for function minimization. The computer journal 7(4), 308-313 (1965)

16. Nguyen, T.V., Baccelli, F., Zhu, K., Subramanian, S., Wu, X.: A performance analysis of csma based broadcast protocol in vanets. pp. 2805-2813. IEEE INFOCOM, Turin, Italy (2013)

17. Noori, H., Olyaei, B.: A novel study on beaconing for vanet-based vehicle to vehicle communication: Probability of beacon delivery in realistic large-scale urban area using 802.11p. pp. 1-6. IEEE SaCoNeT, Paris, France (2013)

18. Shukla, R.K., Trivedi, M., Kumar, M.: On the proficient use of gev distribution: a case study of subtropical monsoon region in india. Annals. Computer Science Series 8 (2010)

19. Stanica, R., Chaput, E., Beylot, A.L.: Broadcast communication in vehicular adhoc network safety applications. pp. 462-466. IEEE CCNC, Lax Vegas, USA (2011)

20. Uchida, M.: Traffic data analysis based on extreme value theory and its applications. pp. 1418-1424. IEEE GLOBECOM, Dallas, USA (2004)

21. Uppoor, S., Fiore, M.: Large-scale urban vehicular mobility for networking research. pp. 62-69. IEEE VNC, Amsterdam, The Netherlands (2011) 